\title{
Bilan et variabilité temporelle des matières en suspension dans le bassin de la Macta : cas du sous bassin versant de Bou-Hanifia (Algérie du nord) Budget and temporal variability of suspended sediment in the Macta basin: the case of Bou-Hanifia sub-basin (northern Algeria)
}

\section{Imène Benstaali et Lahouari Benayada}

Volume 30, numéro 3, 2017

Reçu le 7 avril 2015, accepté le 4 avril 2017

URI : https://id.erudit.org/iderudit/1044247ar

DOI : https://doi.org/10.7202/1044247ar

Aller au sommaire du numéro

Éditeur(s)

Université du Québec - INRS-Eau, Terre et Environnement (INRS-ETE)

ISSN

1718-8598 (numérique)

Découvrir la revue

Citer cet article

Benstaali, I. \& Benayada, L. (2017). Bilan et variabilité temporelle des matières en suspension dans le bassin de la Macta : cas du sous bassin versant de Bou-Hanifia (Algérie du nord). Revue des sciences de l'eau / Journal of Water Science, 30(3), 199-211. https://doi.org/10.7202/1044247ar

\section{Résumé de l'article}

L'intérêt principal des mesures de concentrations des matières en suspension (MES) sur un bassin versant est qu'elles permettent d'établir un bilan moyen des exportations de MES et de déduire un taux d'érosion mécanique à l'échelle du bassin. Cependant, la quantification des flux solides d'un cours d'eau est une tâche délicate, en particulier en zone semi-aride où les crues violentes et de courte durée rendent les prélèvements difficiles. Dans la présente étude, les mesures de débits et de concentrations ont été menées par l'Agence Nationale des Ressources Hydriques sur le bassin versant de Bou-Hanifia, un sous bassin important de la Macta caractérisé par un climat semi-aride et une forte érosion hydrique. Les résultats du bilan témoignent d'une très importante variabilité temporelle du transport solide, tant à l'échelle saisonnière qu'à l'échelle interannuelle. Ceci est lié en grande partie à l'agressivité des précipitations pouvant assurer une très forte et irrégulière mobilisation des MES. À l'échelle du bassin au cours de la période 1993-2006, le flux annuel moyen de MES est estimé à $1,8 \times 10^{6}$ tonnes (t) soit un débit solide spécifique moyen de 343 $\mathrm{t} \cdot \mathrm{a}^{-1} \cdot \mathrm{km}^{-2}$. En effet, les années humides sont les plus exportatrices de sédiments en suspension. Ainsi, la saison d'automne, la plus érosive de l'année, représente $75 \%$ du bilan annuel. L'essentiel des MES est exporté lors des crues de fin d'été - début d'automne, et sont à l'origine de 36 à $94 \%$ des flux de MES transportés annuellement pendant $1 \%$ du temps, soit trois jours par an. 


\section{BILAN ET VARIABILITÉ TEMPORELLE DES MATIÈRES EN SUSPENSION DANS LE BASSIN DE LA MACTA : CAS DU SOUS BASSIN VERSANT DE BOU-HANIFIA (ALGÉRIE DU NORD)}

Budget and temporal variability of suspended sediment in the Macta basin: the case of Bou-Hanifia sub-basin (northern Algeria)

IMÈNE BENSTAALI ${ }^{1,2 *}$ et LAHOUARI BENAYADA ${ }^{1}$

${ }^{1}$ Université des Sciences et Technologie d'Oran, Faculté d'Architecture et de Génie Civil, Département d'Hydraulique, BP 1505 El Manaour, 31000 Oran, Algérie

${ }^{2}$ Université de Mascara, Laboratoire des Sciences et Techniques de l’Eau, Route El Mamounia, 29000 Mascara, Algérie

Reçu le 7 avril 2015, accepté le 4 avril 2017

\section{RÉSUMÉ}

L'intérêt principal des mesures de concentrations des matières en suspension (MES) sur un bassin versant est qu'elles permettent d'établir un bilan moyen des exportations de MES et de déduire un taux d'érosion mécanique à l'échelle du bassin. Cependant, la quantification des flux solides d'un cours d'eau est une tâche délicate, en particulier en zone semi-aride où les crues violentes et de courte durée rendent les prélèvements difficiles. Dans la présente étude, les mesures de débits et de concentrations ont été menées par l'Agence Nationale des Ressources Hydriques sur le bassin versant de Bou-Hanifia, un sous bassin important de la Macta caractérisé par un climat semi-aride et une forte érosion hydrique. Les résultats du bilan témoignent d'une très importante variabilité temporelle du transport solide, tant à l'échelle saisonnière qu'à l'échelle interannuelle. Ceci est lié en grande partie à l'agressivité des précipitations pouvant assurer une très forte et irrégulière mobilisation des MES. À l'échelle du bassin au cours de la période 1993-2006, le flux annuel moyen de MES est estimé à $1,8 \times 10^{6}$ tonnes $(\mathrm{t})$ soit un débit solide spécifique moyen de $343 \mathrm{t} \cdot \mathrm{a}^{-1} \cdot \mathrm{km}^{-2}$. En effet, les années humides sont les plus exportatrices de sédiments en suspension. Ainsi, la saison d'automne, la plus érosive de l'année, représente $75 \%$ du bilan annuel. L'essentiel des MES est exporté lors des crues de fin d'été - début d'automne, et sont à l'origine de 36 à $94 \%$ des flux de MES transportés annuellement pendant $1 \%$ du temps, soit trois jours par an.

Mots-clés : Concentrations des matières en suspension, bassin versant, semi-aride, transport solide, crue, érosion.

\section{ABSTRACT}

The main interest of concentrations measurements of suspended sediment (SS) in a catchment area is to establish an average sediment budget and deduct mechanical erosion rates at the basin scale. However, quantification of SS yield of a river is a delicate task, particularly in semi-arid area where 
the violent floods and short duration make it difficult samples. In the present study, measurements of water discharge and concentrations has been conducted by the National Agency of Water Resources in Bou-Hanifia basin, an important sub-basin of the Macta watershed characterized by a semi arid climate and strong rates of erosion. The sediments budgets results reflect a very strong temporal variability, both at seasonal as interannual scale, of solid transport. This is largely related to the climate aggressivity that can provide a very strong mobilization of SS occurring at varying periods. At the watershed scale during the period 1993-2006, the average sediments budgets is estimated to be $1,8 \times 10^{6}$ tonnes $(\mathrm{t})$ and a degradation of $343 \mathrm{t} \cdot \mathrm{a}^{-1} \cdot \mathrm{km}^{-2}$. Indeed, the wet years are more exporting of SS. Thus, autumn season is the most erosive of the year representing $75 \%$ of the annual budget. In fact, the majority of SS export occurs during flood at the end of summer and the beginning of autumn, and they are at the origin between 36\% and 94\% sediment yield annually transported for $1 \%$ of the time, or three days per year.

\section{Key Words: Concentration of suspended sediment, watershed, semi-arid, sediment transport, flood, erosion.}

\section{INTRODUCTION}

Le transport de sédiments dans les hydrosystèmes est un processus complexe et discontinu à forte variabilité spatiotemporelle, difficile à décrire sous forme d'équation mathématique (TAVARES, 2010). Une bonne compréhension de l'ensemble des facteurs générant et régissant le transport des MES semble donc nécessaire en vue d'identifier les déterminants climatiques ou hydrologiques des MES dans un bassin versant. Le transport solide dans les cours d'eau dépend en grande partie du débit liquide, ce dernier est étroitement lié à la variabilité des précipitations. La relation entre le flux solide et liquide est classiquement représentée par une loi en puissance empirique qui se retrouve entachée d'une forte incertitude (MORGAN, 1995; ASSELMAN, 2000; PICOUET et al., 2001; LAQUIONIE, 2007). Cependant, l'imprécision de cette relation dépend souvent du degré de dispersion des mesures, liée à l'origine et à la disponibilité des particules au cours du temps (VONGVIXAY, 2012).

En Algérie, la forte intensité pluviométrique, la torrentialité des écoulements, la faible densité du couvert végétal et la grande sensibilité des sols à l'érosion constituent les conditions favorables à l'accélération du phénomène de transport solide, qui menace gravement les ressources en eau et en sol des bassins versants. Le transport des sédiments provoque l'envasement des barrages algériens à raison de $3 \%$ chaque année et réduit sensiblement leur capacité de stockage (LEKFIR et al., 2006). Selon BOUDJADJA et al. (2003), alors que 98 ouvrages de retenues des eaux devraient retenir théoriquement une capacité totale de plus de $4,3 \times 10^{6} \mathrm{~m}^{3}$ d'eau, ils ne contiennent en fait que la moitié de ce volume compte tenu de leur envasement. Des études montrent que chaque année $45 \times 10^{6} \mathrm{~m}^{3}$ de matériaux se déposent au fond de 57 grands barrages de l'Algérie, ce qui représente une perte de capacité annuelle égale à $0,7 \%$ de la capacité totale (REMINI et al., 2009). En 2006, le volume total de vase a été estimé à $1,1 \times 10^{9} \mathrm{~m}^{3}$, soit un taux cumulé de comblement de $16 \%$ de la capacité totale de $6,8 \times 10^{9} \mathrm{~m}^{3}$ (REMINI et al., 2009). L'érosion des sols au niveau des bassins versants et le transport solide constituent donc un problème majeur en Algérie, car le taux d'érosion spécifique atteint des valeurs dépassant les $2000 \mathrm{t} \cdot \mathrm{a}^{-1} \cdot \mathrm{km}^{-2}$ (MEGUENNI et REMINI, 2008). Face à la grave pénurie d'eau, à l'envasement rapide des barrages et à la dégradation croissante de la qualité des eaux, il est donc nécessaire de mieux comprendre le fonctionnement général des cours d'eau à travers les bilans de flux hydrique et solide à différentes échelles de temps et les processus qui affectent les matériaux transportés afin de conserver les eaux et les sols, d'éviter l'envasement rapide des barrages et aussi de maintenir une bonne qualité physico-chimique des eaux des rivières.

Dans ce contexte, cette étude s'intéresse à l'analyse de la variabilité du transport des sédiments en suspension à différentes échelles de temps (année et mois) dans le bassin versant de BouHanifia, tributaire du climat semi-aride d'Algérie du Nord. Dans cette région, les conséquences de l'érosion hydrique sont désastreuses et spectaculaires, offrant un paysage nu et sillonné par un ravinement intense, particulièrement dans les régions montagneuses à réseau d'écoulement dense. Les mesures du débit liquide et de la concentration des MES sont réalisées à un pas de temps d'une heure par l'Agence Nationale des Ressources Hydriques sur une période allant de 1993 jusqu'à 2006.

L'objectif de cette étude consiste à faire le bilan des flux de MES dans le bassin versant de Bou-Hanifia et d'analyser la dynamique temporelle à différentes échelles de temps (année, mois et jour). Ce travail identifie également l'impact des crues sur les écoulements liquides et solides dans le bassin.

\section{MATÉRIEL ET MÉTHODES}

\subsection{Site d'étude}

Le bassin versant de Bou-Hanifia, localisé dans la région ouest de l'Algérie, fait partie du grand bassin régional de la Macta (Figure 1). Il est situé dans l'unité physiographique des Hauts Plateaux, entre l'Atlas Tellien au nord et l'Atlas saharien au sud. L'Oued El Hammam draine ce bassin versant et alimente le barrage de Bou-Hanifia, mis en eau en 1948. 

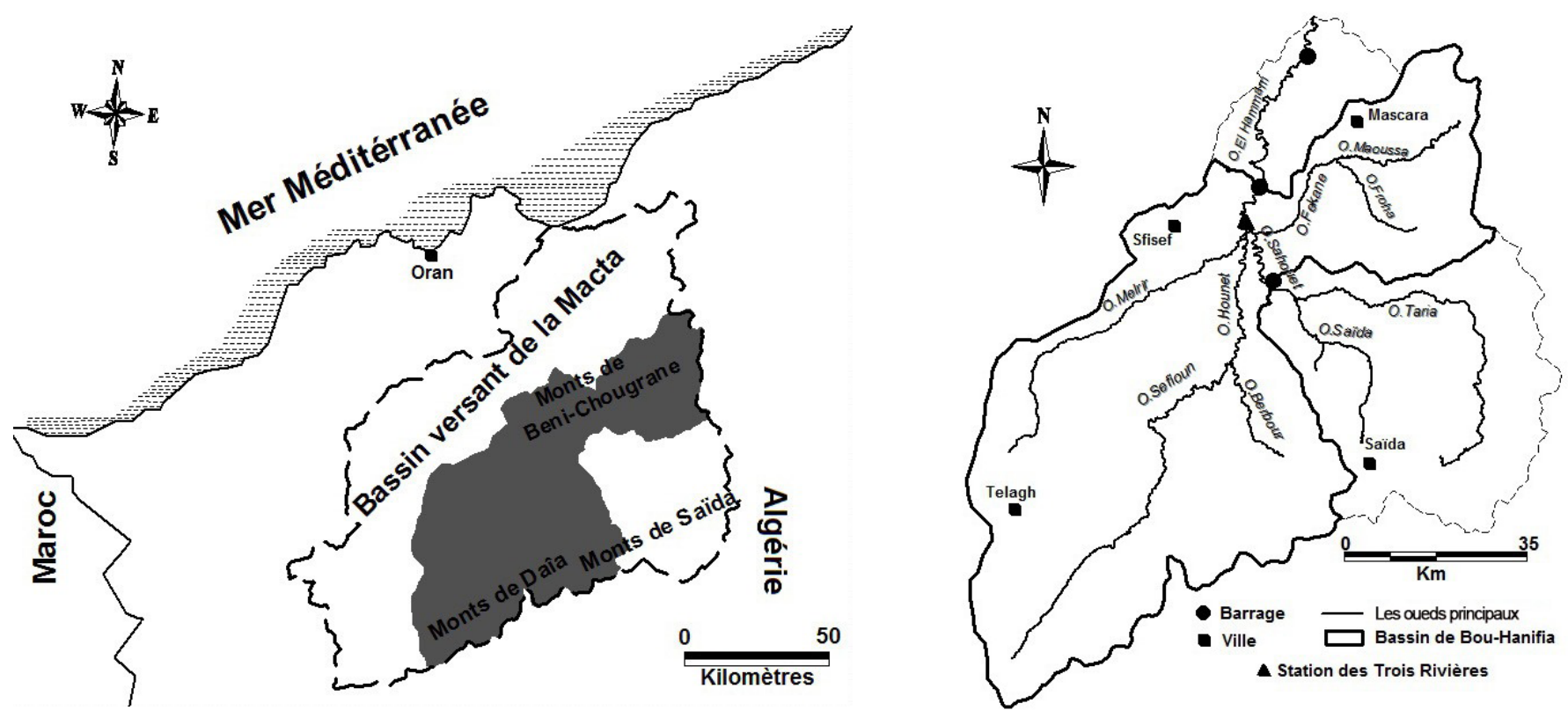

Figure 1. Localisation géographique du bassin versant de Bou-Hanifia. Geographical location of the Bou-Hanifia Basin.

Une série d'oueds aboutissent le long de son bord sud : l'Oued El Hammam se forme dans la vallée des Trois Rivières par la rencontre de l'oued Melrir à l'ouest, l'Oued Hounet au centre et l'Oued Sahouet à l'est (grossi de l'Oued Taria et de l'Oued Saïda), dont les eaux sont recueillies par le barrage Ouizert. L'oued principal a une pente moyenne de $5,7 \mathrm{~m} \cdot \mathrm{km}^{-1}$. La densité de drainage du réseau hydrographique est de $1,7 \mathrm{~km} \cdot \mathrm{km}^{-2}$.

Le bassin de Bou-Hanifia couvre une superficie de $5560 \mathrm{~km}^{2}$ avec une altitude maximale de $1455 \mathrm{~m}$. La partie médiane du bassin versant correspond à des plaines, coteaux ondulés et plateaux. Ces unités sont bordées de collines et de massifs montagneux importants : au nord par les monts de Béni-Chougrane et au sud par les monts de Daïa. Le bassin possède une forme allongée dotée de nombreuses concavités.

Le climat est de type semi-aride, caractérisé par des pluies très violentes en automne provoquant une forte érosion. La pluviométrie moyenne annuelle sur le bassin versant de BouHanifia est estimée à 315 mm sur les années 1973-2011, elle varie de $210 \mathrm{~mm}$ sur les plaines à $470 \mathrm{~mm}$ sur les zones les plus élevées du bassin.

Les roches présentes dans le bassin versant sont toutes d'origine sédimentaire. Les dépôts quaternaires sont localisés principalement le long des oueds. Le bassin est occupé par les formations géologiques suivantes : des roches sédimentaires indifférenciées $(3 \%)$, des brèches et conglomérats $(14 \%)$, des marnes $(30 \%)$, des calcaires $(21 \%)$, des grés $(9 \%)$, des argiles $(1 \%)$ et des dépôts meubles sur environ $22 \%$ de la surface totale du bassin (Figure 2).
L'activité du bassin versant de Bou-Hanifia est principalement dominée par l'agriculture. Elle est basée sur les céréales, les plantes fourragères et oléagineuses, le maraîchage, les arbres fruitiers, l'olivier, le raisin et l'élevage. Toutefois, les grandes cultures sont temporaires, discontinues et ne protègent pas efficacement le sol. Le milieu naturel représente environ $50 \%$ de la surface du bassin, où dominent la forêt, les maquis et les parcours. Les massifs forestiers ont diminué ces dernières années suite à l'action anthropique et aux incendies, ce qui a favorisé les risques d'érosion et de ruissellement à l'échelle du bassin. Ces massifs forestiers se concentrent sur les versants montagneux et occupent près du quart du bassin. Les maquis, matorrals et steppes se présentent sous forme de mosaïque. Ils sont concentrés aux bords des massifs forestiers, ainsi que dans la pointe sud-ouest du bassin versant.

\subsection{Données utilisées}

La banque de données utilisée est constituée des mesures instantanées de débits liquides et de concentration des MES effectuées par l'Agence Nationale des Ressources Hydriques d'Oran à la station des Trois Rivières, seule station située juste en amont du barrage de Bou-Hanifia et à la confluence de trois affluents principaux. Cette base contient 13 années de données de 1993 à 2006. Nous disposons également des séries de débits liquides journaliers et de précipitations mensuelles sur la même période. Les débits solides ont été obtenus à partir des valeurs instantanées des débits liquides $(Q)$, exprimées en $\mathrm{m}^{3} \cdot \mathrm{s}^{-1}$, et de la concentration des MES $\left(\mathrm{g} \cdot \mathrm{L}^{-1}\right)$. Pour la mesure de concentration des MES, à chaque mesure de débit, on prélève au moyen d'un 


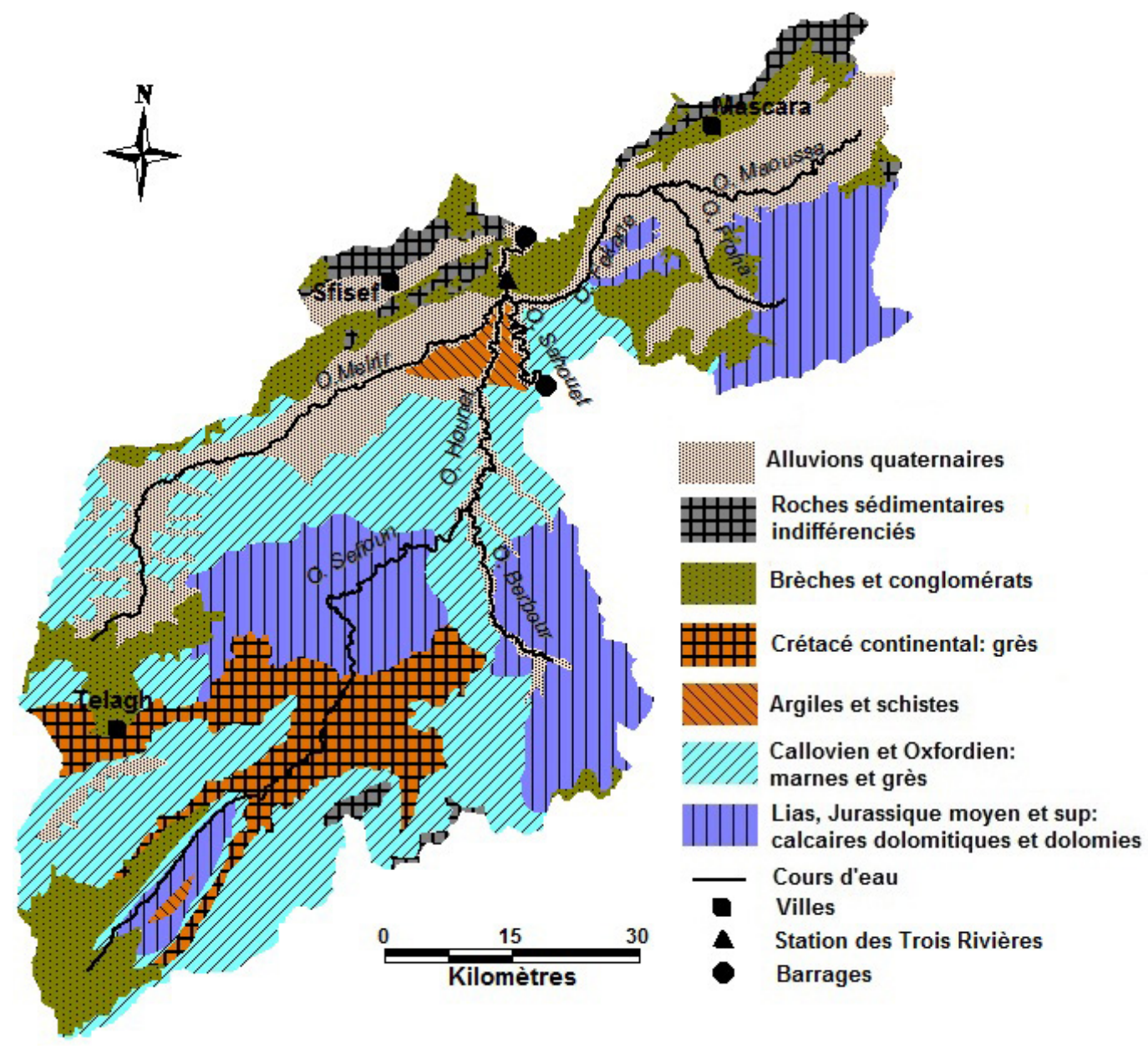

Figure 2. Carte géologique du bassin versant de Bou-Hanifia.

Geological map of the Bou-Hanifia Basin.

flacon de $50 \mathrm{cL}$ un échantillon d'eau turbide sur la rive à la surface de l'oued. Les sédiments recueillis sur papier filtre sont ensuite séchés à l'étuve pendant 30 min à une température de $105^{\circ} \mathrm{C}$. On détermine ensuite la charge correspondant à un litre d'eau prélevée, ce qui établit la concentration, exprimée en $\mathrm{g} \cdot \mathrm{L}^{-1}$. La fréquence de prise des mesures dépend de la variation de la hauteur d'eau dans l'oued. En période de crue, les prises sont intensifiées jusqu'à des intervalles de temps d'une heure ou même 30 min en fonction de la vitesse de l'augmentation des débits liquides. En période d'écoulement normal ou en période d'étiage, on se contente d'une prise quotidienne effectuée généralement à $12 \mathrm{~h}$.

Le débit solide est calculé par l'équation suivante :

$$
Q_{s}=C_{s} Q_{l}
$$

où $Q_{\text {s }}$ est le débit solide en suspension $\left(\mathrm{kg} \cdot \mathrm{s}^{-1}\right), Q_{l}$ est le débit liquide $\left(\mathrm{m}^{3} \cdot \mathrm{s}^{-1}\right)$ et $C_{s}$ est la concentration $\left(\mathrm{g} \cdot \mathrm{L}^{-1}\right)$.
Dans le cadre de ce travail, la quantité de MES qui transite à un instant donné dans un cours d'eau sur le pas de temps de mesure d'une période donnée (heure, journée, mois ou année), est calculée pendant un intervalle de temps séparant deux prélèvements consécutifs par la formule suivante :

$$
\begin{aligned}
F_{s}(T) & =\sum_{j=1}^{j=n} \Delta t_{j} Q m_{j} C m_{j} 10^{-3} \\
& =\sum_{j=1}^{j=n}\left(\frac{\left(C_{j} Q_{j}\right)+\left(C_{j+1} Q_{j+1}\right)}{2}\right)\left(t_{j+1}-t_{j}\right) 10^{-3}
\end{aligned}
$$

où $F_{s}(T)$ représente la quantité ou le flux de $\operatorname{MES}(\mathrm{t}), \Delta t_{j}$ est la durée séparant deux prélèvements consécutifs en secondes, $Q m_{j}\left(\mathrm{~m}^{3} \cdot \mathrm{s}^{-1}\right)$ et $C m_{j}\left(\mathrm{~g} \cdot \mathrm{L}^{-1}\right)$ sont les débits liquides moyens et les concentrations des MES moyennes pendant l'intervalle de temps $\Delta t$, respectivement, et $n$ est le nombre de prélèvements effectués sur la période considérée. 
Le débit en MES spécifique est évalué par le rapport des flux de MES à la superficie totale du bassin versant. Il est calculé par :

$$
F_{s s}=\frac{F_{s}}{S}
$$

où $F_{s s}$ est le débit en MES spécifique ou la dégradation spécifique $\left(\mathrm{t} \cdot \mathrm{a}^{-1} \cdot \mathrm{km}^{-2}\right), F_{s}$ est le flux de MES annuel, mensuel ou saisonnier (t), et $S$ est la superficie du bassin versant $\left(\mathrm{km}^{2}\right)$.

\section{RÉSULTATS ET DISCUSSION}

\subsection{Bilan annuel et variabilité interannuelle des précipitations, des flux liquides et des flux de MES}

En moyenne, le flux annuel de MES dans le bassin versant calculé à la station des Trois Rivières s'élève à $1,8 \times 10^{6} \mathrm{t} \cdot \mathrm{a}^{-1}$. Si on admet que la surface drainée par l'Oued El Hammam à cette station est de $5322 \mathrm{~km}^{2}$, la dégradation spécifique est très différente d'une année à l'autre. En effet, elle varie de 32,7 à $1170 \mathrm{t} \cdot \mathrm{a}^{-1} \cdot \mathrm{km}^{-2}$, soit une érosion hydrique moyenne de l'ordre de $343 \mathrm{t} \cdot \mathrm{a}^{-1} \cdot \mathrm{km}^{-2}$ (Tableau 1$)$. Cette valeur de l'érosion hydrique obtenue est très cohérente avec les résultats obtenus dans le bassin de l'Oued Moulouya au Maroc $\left(336 \mathrm{t} \cdot \mathrm{a}^{-1} \cdot \mathrm{km}^{-2}\right.$; MERZOUKI, 1992) et au microbassin de Tebaga en Tunisie centrale (318 t.a-1 $\mathrm{km}^{-2}$; BERGAOUI et al., 1998). En outre, elle est tout à fait dans la gamme des valeurs estimées pour les bassins versants du Maghreb (269 à $2569 \mathrm{t} \cdot \mathrm{a}^{-1} \cdot \mathrm{km}^{-2}$; HEUSCH et MILLIES-LACROIX, 1971). En revanche, la valeur est largement inférieure aux taux d'érosion proposés par WALLING (1984), compris entre 1000 et $5000 \mathrm{t} \cdot \mathrm{a}^{-1} \cdot \mathrm{km}^{-2}$. Elle est également au-dessous des estimations réalisées en Algérie, par exemple le bassin de l'Oued Agrioun (7 $200 \mathrm{t} \cdot \mathrm{a}^{-1} \cdot \mathrm{km}^{-2}$; PROBST et AMIOTTE-SUCHET, 1992) et le bassin de la Haute Tafna (1 $120 \mathrm{t} \cdot \mathrm{a}^{-1} \cdot \mathrm{km}^{-2}$; MEGNOUNIF et al., 2003), au Maroc (5 $900 \mathrm{t} \cdot \mathrm{a}^{-1} \cdot \mathrm{km}^{-2}$ [LAHLOU, 1988]; $2142 \mathrm{t} \cdot \mathrm{a}^{-1} \cdot \mathrm{km}^{-2}$ [SIBARI et al., 2001]; $750 \mathrm{t} \cdot \mathrm{a}^{-1} \cdot \mathrm{km}^{-2}$ [SNOUSSI, 1988]) et à Santiago, Cap Vert, dans le bassin de Longueira (4 $266 \mathrm{t} \cdot \mathrm{a}^{-1} \cdot \mathrm{km}^{-2}$; TAVARES, 2010). Par contre, les résultats trouvés sont nettement supérieurs aux taux moyens à l'échelle globale estimés à environ à $150 \mathrm{t} \cdot \mathrm{a}^{-1} \cdot \mathrm{km}^{-2}$ (LUDWIG et PROBST, 1998; MILLIMAN et MEADE, 1983; WALLING et WEBB, 1983). Ils sont largement au-dessus des valeurs de la dégradation estimées pour le bassin de l'Oued Mouillah (126 $\mathrm{t} \cdot \mathrm{a}^{-1} \cdot \mathrm{km}^{-2}$; TERFOUS et al., 2001), sur l'Oued Sikkak et l'Oued Isser (170 et $180 \mathrm{t} \cdot \mathrm{a}^{-1} \cdot \mathrm{km}^{-2}$; BOUANANI, 2004), sur l'Oued Abd (136 t: $\mathrm{a}^{-1} \cdot \mathrm{km}^{-2}$; ACHITE et OUILLON, 2007), dans le bassin versant de l'Oued Leham, Algérie orientale (104 $\mathrm{t} \cdot \mathrm{a}^{-1} \cdot \mathrm{km}^{-2}$; BOUROUBA, 1998) et dans les bassins de
Tableau 1. Bilan et variabilité des précipitations $(P)$, des flux liquides $(F)$, des flux de matières en suspension, MES $\left(F_{s}\right)$ et des MES spécifiques $\left(F_{s s}\right)$.

Table 1. Budget and variability of rainfall $(P)$, stream flow $\left(F_{p}\right)$, sediment yield $(F)$ and specific suspended sediment $\left(F_{s}\right)$.

\begin{tabular}{lcccc}
\hline Année & $\begin{array}{c}\boldsymbol{P} \\
(\mathbf{m m})\end{array}$ & $\begin{array}{c}\boldsymbol{F}_{\boldsymbol{l}} \\
\left(\mathbf{1 0}^{\mathbf{6}} \mathbf{m}^{\mathbf{3}}\right)\end{array}$ & $\begin{array}{c}\boldsymbol{F}_{\boldsymbol{S}} \\
\left(\mathbf{1 0 ^ { 5 }} \mathbf{t}\right)\end{array}$ & $\begin{array}{c}\boldsymbol{F}_{\boldsymbol{s s}} \\
\left(\mathbf{t} \cdot \mathbf{k m}^{-\mathbf{2}}\right)\end{array}$ \\
\hline $1993-1994$ & 248,6 & 26,71 & 4,40 & 82,7 \\
$1994-1995$ & 319,4 & 125,83 & 62,25 & 1169,9 \\
$1995-1996$ & 427,1 & 71,37 & 8,45 & 158,8 \\
$1996-1997$ & 205,9 & 90,90 & 21,21 & 398,5 \\
$1997-1998$ & 287,9 & 98,56 & 11,37 & 213,6 \\
$1998-1999$ & 204,1 & 39,84 & 5,27 & 99,0 \\
$1999-2000$ & 223,1 & 43,85 & 15,68 & 294,8 \\
$2000-2001$ & 295,5 & 97,58 & 39,99 & 751,4 \\
$2001-2002$ & 301,3 & 99,56 & 43,38 & 815,1 \\
$2002-2003$ & 344,5 & 41,25 & 3,54 & 66,5 \\
$2003-2004$ & 325,8 & 56,20 & 11,26 & 211,6 \\
2004-2005 & 268,6 & 41,44 & 8,68 & 163,1 \\
2005-2006 & 348,7 & 19,31 & 1,74 & 32,7 \\
& & & 18,25 & 342,9 \\
Moyenne & 292,3 & 65,57 & 18,08 & - \\
Écart-type & 63,61 & 33,70 & 1,02 & - \\
Cv ${ }^{\mathrm{a}}$ & 0,22 & 0,51 & &
\end{tabular}

${ }^{\mathrm{a}}$ Coefficient de variation

Grande et de Godim à Santiago, Cap Vert, avec $157 \mathrm{t} \cdot \mathrm{a}^{-1} \cdot \mathrm{km}^{-2}$ et $10,2 \mathrm{t} \cdot \mathrm{a}^{-1} \cdot \mathrm{km}^{-2}$, respectivement (TAVARES, 2010).

D'après le tableau 1 et la figure 3 , on note une concordance entre les précipitations et les débits en MES spécifiques avec sept valeurs dont quatre valeurs correspondent aux années déficitaires (1993-1994, 1998-1999, 1999-2000 et 20042005 ) et trois valeurs correspondent aux années excédentaires (1994-1995, 2000-2001 et 2001-2002). Par contre, le nombre s'élève à 12 valeurs pour les flux liquide et de MES spécifiques dont sept valeurs correspondent aux années déficitaires (19931994, 1998-1999, 1999-2000, 2002-2003, 2003-2004, 2004-2005 et 2005-2006) et cinq valeurs correspondent aux années excédentaires (1994-1995, 1995-1996, 1996-1997, 2000-2001 et 2001-2002). Ainsi, les débits liquides et les débits en MES spécifiques ne suivent pas la même tendance que les précipitations. Il convient toutefois de noter que cette constatation a déjà été signalée auparavant par de nombreux auteurs (EL MAHI et al., 2012; GHENIM et al., 2008; VONGVIXAY, 2012). De ce fait, l'année la plus pluvieuse (1995) correspond à un écoulement légèrement supérieur à la valeur moyenne annuelle, par contre les flux liquide et de MES sont nettement plus élevés pour l'année 1994, alors que la pluviosité est à peine supérieure à la moyenne.

D'une manière générale, l'année la plus humide n’implique pas forcément que cette année soit la plus contributive en flux hydrique et productive en MES et vice versa. La discordance dans le temps entre le maximum annuel de précipitation, 


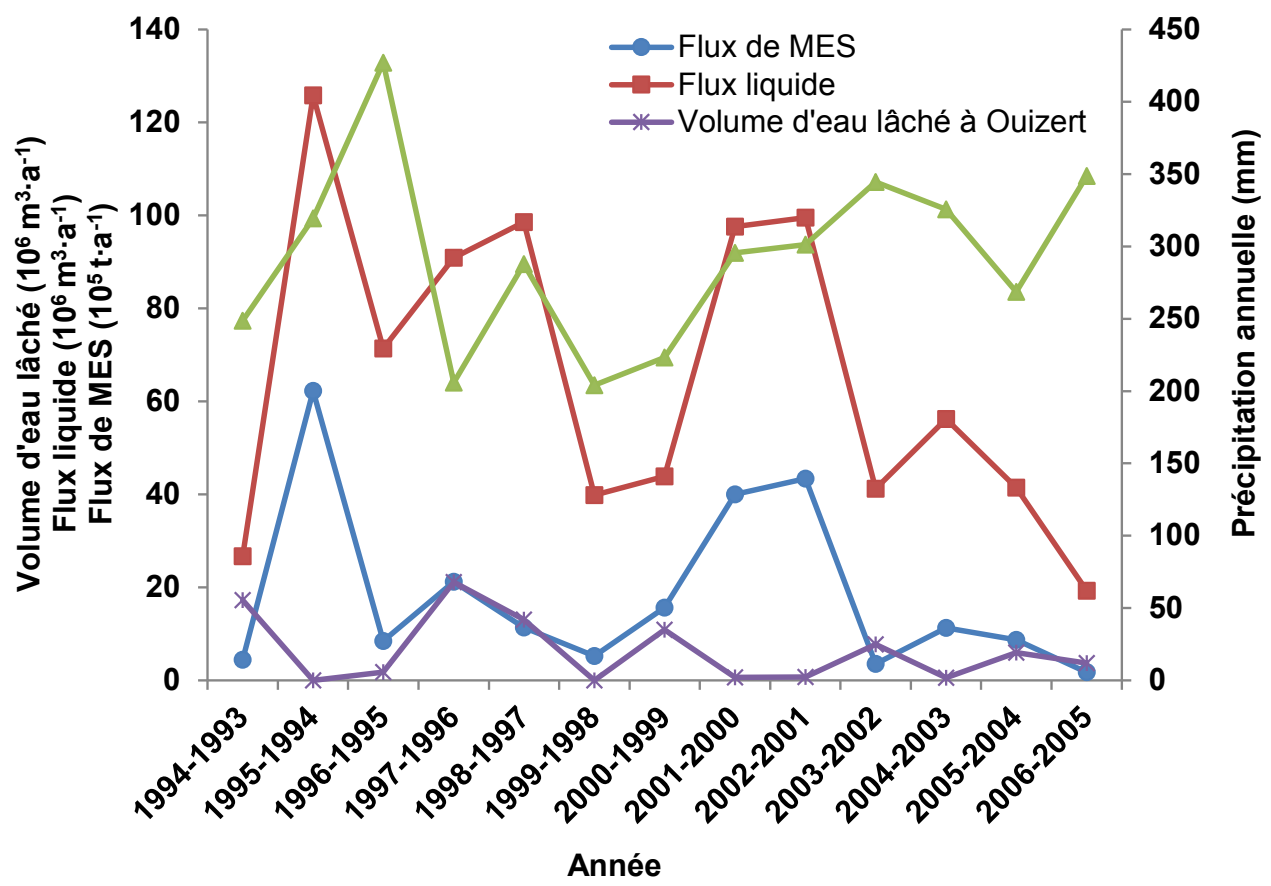

Figure 3. Variabilité interannuelle des précipitations, des flux liquides, des flux de matières en suspension (MES) à la station des Trois Rivières dans le bassin versant de BouHanifia et les volumes d'eau lâchés du barrage Ouizert (1993-2006).

Interannual variability of rainfall, stream flow, suspended sediment yields at the Three Rivers gauging site in the Bou-Hanifia Basin and the volumes of water released from the Ouizert dam (1993-2006).

des flux hydrique et de MES montre bien que la dynamique de variation des MES n'est pas toujours contrôlée par la pluviométrie, mais plutôt par d'autres facteurs tels que :

i. les lâchers du barrage Ouizert, destinés à soutenir le débit du cours d'eau d'Oued Sahouet, peuvent influencer les débits et augmenter les risques d'érosion des berges. L'évolution annuelle des flux liquides et des volumes d'eau lâchers par le barrage Ouizert (Figure 3) montre de façon générale que les lâchers ne sont pas proportionnels aux flux liquides, le volume total des lâchers est estimé à $83,6 \times 10^{6} \mathrm{~m}^{3}$, soit une contribution de $9 \%$ de flux liquide total. Ceci confirme que les lâchers du barrage n'ont pas d'effet sensible sur les flux d'eau transités annuellement par l'Oued El Hammam à Trois Rivières;

ii. l'état des sols cultivés qui peut varier au sein d'une année et d'une année sur l'autre;

iii. la présence de relief karstique (monts de Saïda et Daïa) permet aux circulations d'eaux souterraines de soutenir l'écoulement superficiel (rétention et restitution souterraine);

iv. la disponibilité des matériaux dans les zones proches du cours d'eau ou dans le cours d'eau lui-même facilement mobilisables même pour de faibles débits. En effet, les sédiments transportés par l'oued et ses affluents proviennent en forte proportion des zones d'apport, très proches du site de mesure, constituées par les formations argileuses et marneuses des monts de Beni-Chougrane (EL MAHI et al., 2012).

La variabilité interannuelle des flux liquide et de MES montre une grande irrégularité, liée à l'irrégularité des précipitations. En effet, les valeurs du coefficient de variation sont très élevées et dépassent l'unité pour les flux de MES (Tableau 1, Figure 3).

\subsection{Variabilité saisonnière et mensuelle}

$\mathrm{Au}$ pas de temps saisonnier, la dégradation spécifique moyenne du bassin versant en automne et en été, représente $75,7 \%$ et $16,1 \%$ de la dégradation annuelle du sol respectivement. Les flux de MES observés en saison d'automne proviennent d'orages survenus sur le bassin, engendrant des crues très chargées où les concentrations instantanées observées atteignent $239,4 \mathrm{~g} \cdot \mathrm{L}^{-1}$ durant la période d'observation. Au cours de la saison sèche, les précipitations sont généralement faibles, voire nulles, mais quelques crues estivales produites par des orages localisés contribuent à augmenter le transport sédimentaire de manière significative. En hiver $(2,6 \%)$ et au printemps $(5,6 \%)$ la charge en suspension n'augmente pas proportionnellement aux précipitations si bien que le flux saisonnier reste inférieur à celui mesuré en automne malgré 
une hausse très significative des totaux pluviométriques. Le résultat obtenu pour d'autres bassins en Algérie montre que l'essentiel de l'érosion est observé en début d'automne et en fin de printemps (ACHITE et MEDDI, 2005; ACHITE et OUILLON, 2007; MEGNOUNIF et al., 2003; HASBAIA et al., 2012).

À l'échelle mensuelle, l'activité érosive du bassin met en évidence deux périodes bien distinctes (Figures $4 \mathrm{a}$ à d) :

- Une période de faible érosion allant de décembre à juillet. Elle correspond à la fois à : i) l'augmentation progressive des précipitations de la saison d'hiver. Lors de cette saison l'écoulement et le transport en MES sont faibles, les précipitations assurent sans doute la recharge des nappes souterraines jusqu'au début du mois de juin; ii) la diminution des précipitations, de l'écoulement et du transport en MES à partir de la fin du printemps. À l'exception le mois de juin, on observe une légère augmentation du débit liquide malgré sa faible pluviométrie, due principalement à la saturation hydrique des sols du bassin qui favorise la genèse rapide des crues survenues.

- Une période de forte érosion avec trois mois : août, septembre et octobre. Elle est caractérisée par des précipitations intenses capables de provoquer un fort ruissellement superficiel, qui engendre une érosion assez importante au niveau de la couche superficielle du sol. En outre, ces trois mois représentent les mois pendant lesquels la production de MES est la plus forte avec respectivement $0,45 \times 10^{6}, 0,76 \times 10^{6}$ et $0,21 \times 10^{6} \mathrm{t}^{-\mathrm{a}^{-1}}$ soit $24,5 \%$, $43,4 \%$ et $11 \%$ du bilan annuel. La production est environ 50 fois plus faible en décembre et en janvier avec respectivement $0,01 \times 10^{6}$ et $0,02 \times 10^{6} \mathrm{t} \cdot \mathrm{a}^{-1}$ soit $0,6 \%$ et $0,8 \%$ du bilan annuel.

Entre les deux périodes se trouve le mois de novembre avec 7,7 \% de flux en MES annuel. Les fortes précipitations du mois de novembre donnent lieu à un écoulement superficiel moins important qu'au mois d'octobre. Ceci est dû au fait que le mois d'octobre marque la transition entre la longue période sèche et la période humide de l'année où les précipitations, souvent agressives et intenses, tombent sur un sol asséché et mal protégé par la végétation (CHEBBANI et al., 1999). Ce qui fait que les flux liquides sont les plus élevés et transportent des quantités importantes de sédiments.

Durant la période d'étude (1993-2006), les débits liquides spécifiques mensuels montrent une tendance d'évolution assez similaire à celles des flux spécifiques de MES mensuels et des concentrations en MES. Les débits mensuels liquide et de MES sont plus élevés en automne (octobre) et plus faibles en hiver (décembre) (Figures 4b, c et d). Ceci peut s'expliquer par le fait que la plupart des crues importantes ont été enregistrées durant la saison d'automne.
Pour une vision plus détaillée, l'analyse des débits journaliers des années hydrologiques 1994-1995, 19961997, 1997-1998, 1999-2000, 2000-2001, 2001-2002 et 2002-2003 révèle des phénomènes majeurs qui ont perturbé le régime saisonnier de l'oued durant la période d'étude : la première crue du 29 septembre 1994 (débit moyen journalier $199,86 \mathrm{~m}^{3} \cdot \mathrm{s}^{-1}$, débit instantané $\left.805 \mathrm{~m}^{3} \cdot \mathrm{s}^{-1}\right)$ a évacué $34 \%$ de flux liquide annuel et $70 \%$ de flux annuel en MES, alors que, la crue du 11 (débit moyen journalier $129,59 \mathrm{~m}^{3} \cdot \mathrm{s}^{-1}$, débit instantané $1168,4 \mathrm{~m}^{3} \cdot \mathrm{s}^{-1}$,) et 17 octobre (débit moyen journalier $258,33 \mathrm{~m}^{3} \cdot \mathrm{s}^{-1}$, débit instantané $2335 \mathrm{~m}^{3} \cdot \mathrm{s}^{-1}$,) ont généré près de $40 \%$ de flux liquide annuel et $27 \%$ de flux annuel en MES. Le flux liquide annuel de $39 \%$ et $67 \%$ de flux annuel en MES ont été exportés au cours de la crue du 25 août 1997 (débit moyen journalier $371,15 \mathrm{~m}^{3} \cdot \mathrm{s}^{-1}$, débit instantané $1622,40 \mathrm{~m}^{3} \cdot \mathrm{s}^{-1}$ ). La crue du 28 juin 1998 (débit moyen journalier $43,30 \mathrm{~m}^{3} \cdot \mathrm{s}^{-1}$, débit instantané $58 \mathrm{~m}^{3} \cdot \mathrm{s}^{-1}$ ) mobilise peu de sédiments avec $14 \%$ de flux liquide annuel. Ainsi, en 2000, la crue d'octobre (débit moyen journalier $468,47 \mathrm{~m}^{3} \cdot \mathrm{s}^{-1}$, débit instantané $\left.2360 \mathrm{~m}^{3} \cdot \mathrm{s}^{-1}\right)$ a apporté à elle seule plus de $62 \%$ du volume d'eau annuel et $93 \%$ de flux annuel en MES. Lors de l'événement du 28 août 2001 (débit moyen journalier $20,8 \mathrm{~m}^{3} \cdot \mathrm{s}^{-1}$, débit instantané $21,04 \mathrm{~m}^{3} \cdot \mathrm{s}^{-1}$ ), environ $11 \%$ de flux liquide annuel et $3 \%$ de flux annuels en MES ont transité à la station des Trois Rivières, alors que $38 \%$ de flux liquide annuel et $76 \%$ de MES annuel ont été transportés durant la crue d'octobre 2001 (débit moyen journalier 396,85 $\mathrm{m}^{3} \cdot \mathrm{s}^{-1}$, débit instantané $1450 \mathrm{~m}^{3} \cdot \mathrm{s}^{-1}$ ). Les deux crues du 8 (débit moyen journalier $35 \mathrm{~m}^{3} \cdot \mathrm{s}^{-1}$, débit instantané $54 \mathrm{~m}^{3} \cdot \mathrm{s}^{-1}$ ) et 24 août 2002 (débit moyen journalier $131,53 \mathrm{~m}^{3} \cdot \mathrm{s}^{-1}$, débit instantané $650 \mathrm{~m}^{3} \cdot \mathrm{s}^{-1}$ ), représentent $17 \%$ de flux liquide annuel et $13 \%$ de flux annuel en MES. L'été 2003 a connu deux crues survenues en août, la plus importante (débit moyen journalier $95,10 \mathrm{~m}^{3} \cdot \mathrm{s}^{-1}$, débit instantané $\left.265 \mathrm{~m}^{3} \cdot \mathrm{s}^{-1}\right)$ produit $52 \%$ de MES avec $28 \%$ du volume d'eau annuel.

L'examen des données journalières du volume d'eau lâché par le barrage Ouizert confirme qu'à Trois Rivières, les volumes d'eau écoulés par l'Oued El Hammam lors des crues sus- citées ne sont pas influencés par les lâchers d'eau du barrage. Tandis que, le débit de l'oued a connu une augmentation lors des crues de juin 1997, juillet 1998 et juillet 2003, alors que le pluviomètre de la station n'a enregistré aucune pluie. Ainsi, les données pluviométriques des stations situées à proximité et même de toutes les stations du bassin n'enregistrent aucune précipitation. Cette hausse de débit est expliquée par les lâchers d'eau du barrage de Ouizert et non pas due aux éventuelles erreurs de mesure. Donc, la mise en place de ce dernier a induit une modification du régime hydrologique de l'Oued El Hammam, marqué par les lâchers d'eau. Afin de mieux apprécier le degré d'influence du barrage sur l'écoulement, on a comparé les deux régimes hydrologiques de l'oued : le régime naturel (sans lâchers d'eau) et le régime influencé (y compris les lâchers d'eau). 

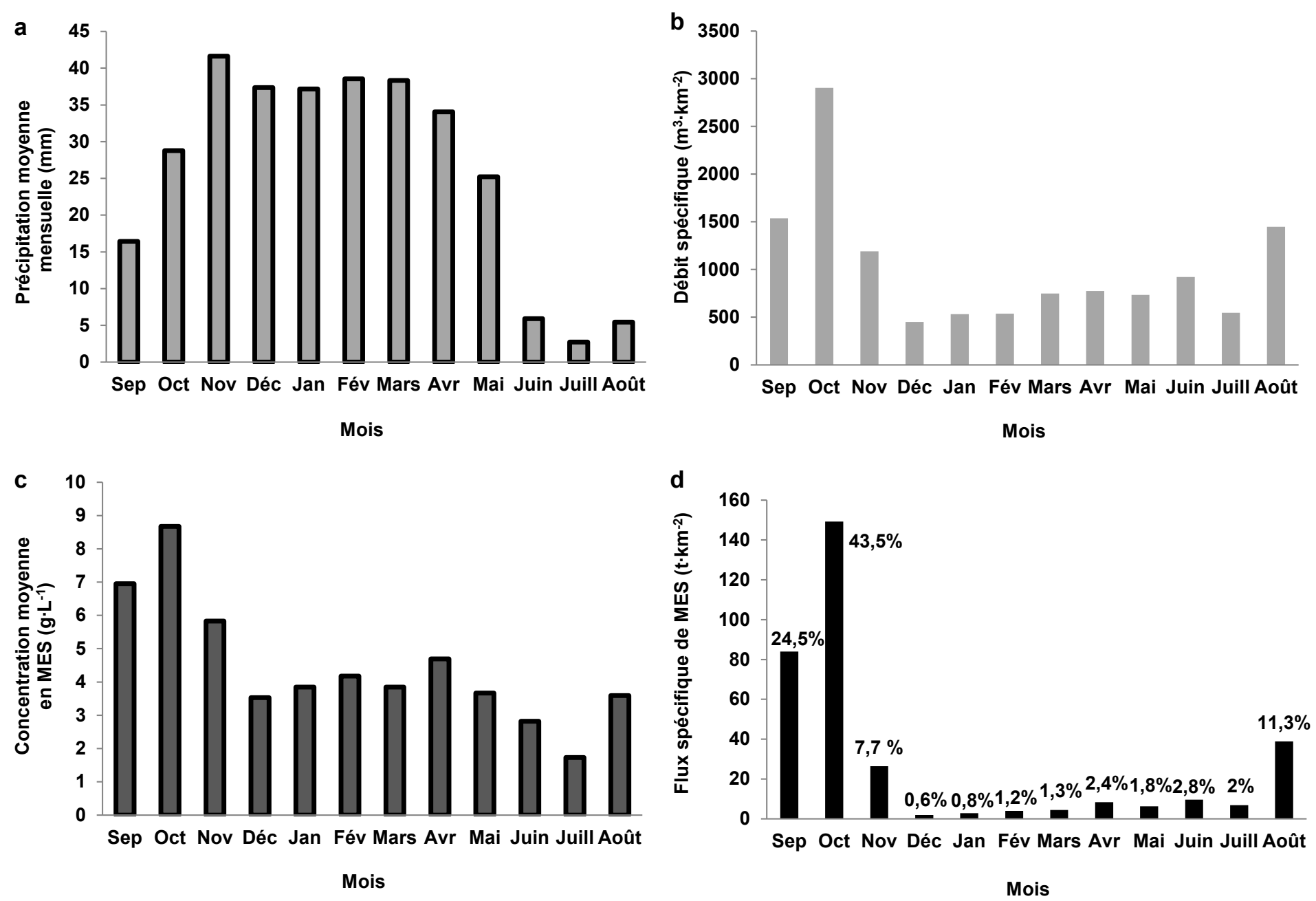

Figure 4. Variabilité mensuelle des : a) précipitations, b) débits liquides spécifiques, c) concentrations en matières en suspension (MES) et d) MES spécifiques durant la période d'étude 1993-2006.

Monthly variability of: a) rainfall, b) specific stream flow, c) concentrations of suspended sediment (SS) and d) specific SS during the study period 1993-2006.

Dans les deux régimes, les débits moyens mensuels les plus soutenus sont observés au cours des mois de septembre, octobre, novembre et août (Figure 5). Aussi, le maximum du débit mensuel est situé au mois d'octobre, alors qu’en régime naturel, le minimum est atteint en juillet avec un débit moyen de l'ordre de $0,5 \mathrm{~m}^{3} \cdot \mathrm{s}^{-1}$. Ce minimum est assez précoce en régime influencé (artificiel) où il est observé au mois de décembre.

Sous le régime artificiel (Figure 5), la période marque une perturbation du régime de l'oued au cours des mois d'avril, juin et juillet. Durant ces trois mois, on assiste à une augmentation bien marquée du débit avec respectivement $37 \%$, $38 \%$ et $55 \%$. Par ailleurs, on constate une augmentation modérée de $10 \%$ du débit de mois d'août et elle est très modérée en octobre (3\%). Le reste de l'année, la contribution mensuelle des lâchers d'eau du barrage Ouizert est nettement faible, voire nulle, elle varie d'une année à l'autre et en fonction des besoins en irrigation.

\subsection{Temps de transfert des MES sur le bassin versant}

\subsubsection{Pourcentage du flux de MES en fonction du temps}

En cumulant les flux de MES (rangés par ordre décroissant) en fonction du temps, des régimes de transports différents peuvent être clairement identifiés (MEYBECK et al., 2003). La courbe de durée représente le pourcentage du flux de MES qui transite en fonction du pourcentage du temps durant la période de mesure (Figure 6). Ces courbes permettent de confirmer et de quantifier le caractère épisodique du transport en MES à l'échelle du bassin versant étudié.

À une durée correspondant à $1 \%$ du temps (soit trois jours par an), les années qui transitent le plus de sédiments sont : 1994-1995, 1999-2000, 2000-2001, 2001-2002 et 20042005 avec plus de $80 \%$ des flux de MES pour des volumes d'eau relativement importants de $46 \%, 27 \%, 62 \%, 60 \%$ et $56 \%$, respectivement. Suivies par les années 1996-1997, 20022003 et 2003-2004 où les MES transportés varient entre $50 \%$ 


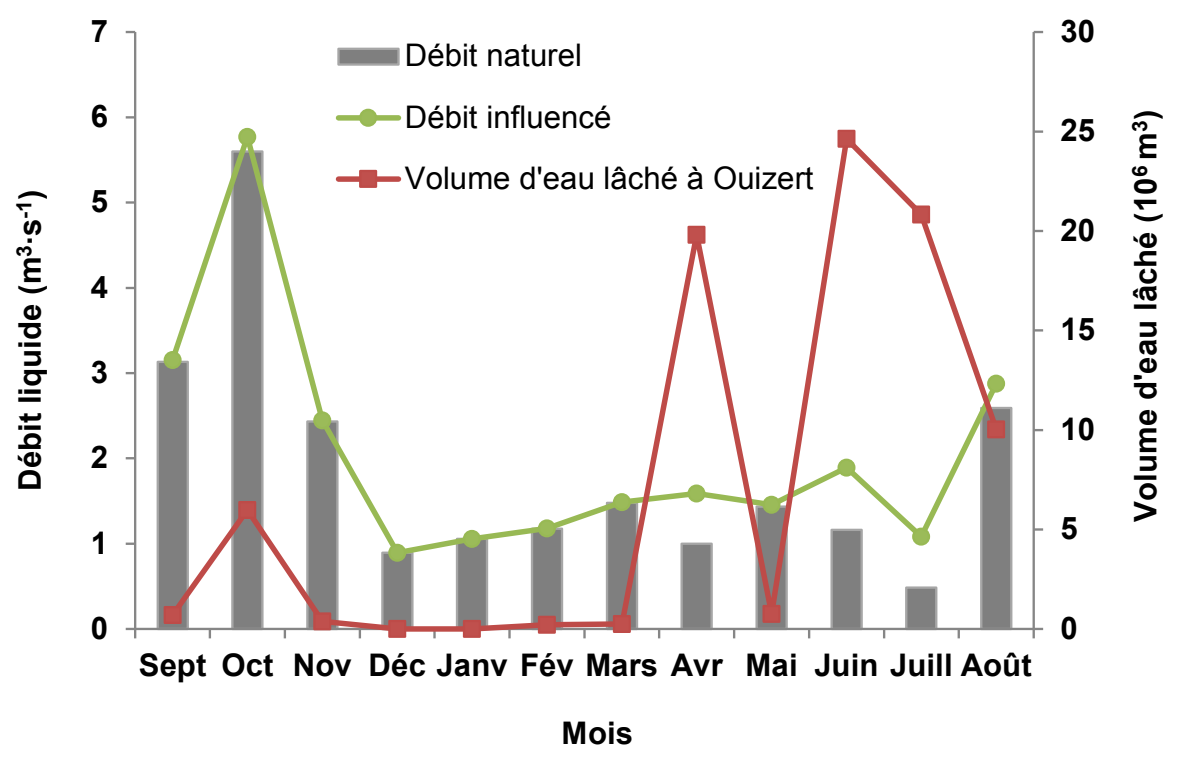

Figure 5. Variation des volumes d'eau lâchés, des débits naturels et influencés à la station des Trois Rivières (1993-2006).

Variation of water volumes released, natural and influenced flows at the Three Rivers gauging site (1993-2006).

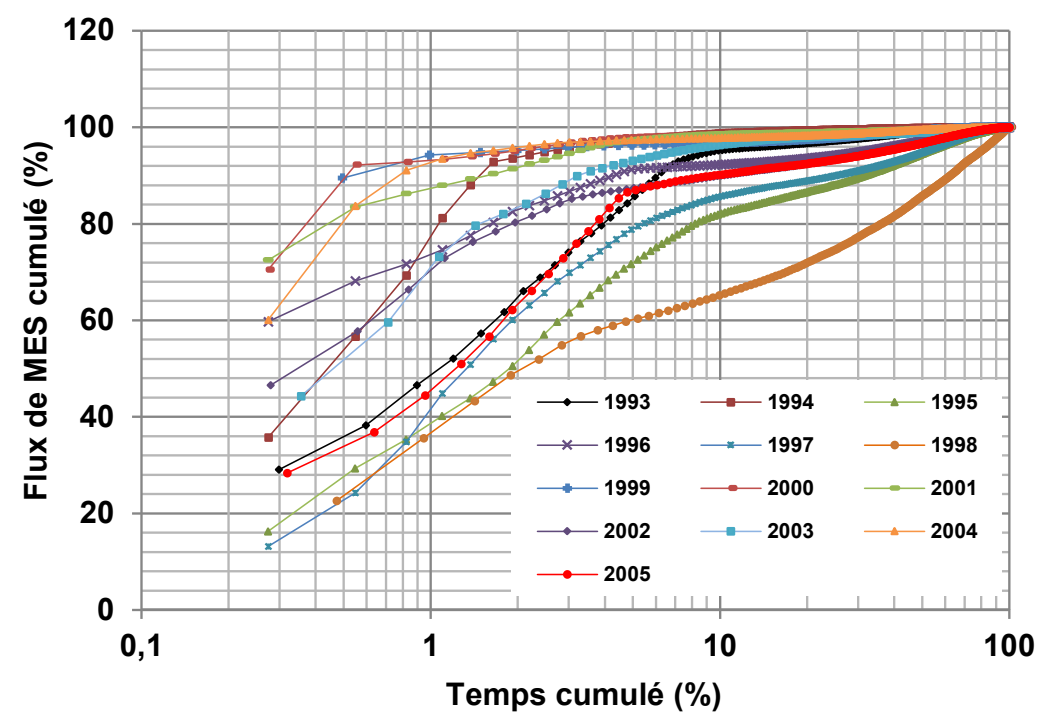

Figure 6. Courbes de durée - flux de matières en suspension (MES) cumulés (classés par ordre décroissant) en fonction du temps cumulé.

Duration curves - cumulative suspended sediment (SS) yields (ranked in descending order) as a function of cumulative time.

et $80 \%$ avec seulement $46 \%, 32 \%$ et $38 \%$ des flux liquides. Entre $36 \%$ et $50 \%$ de sédiments sont transportés par $30 \%$, $17 \%, 24 \%, 17 \%$ et $22 \%$ des flux liquides, respectivement pour les années 1993-1994, 1995-1996, 1997-1998, 19981999 et 2005-2006 (Figure 6).

À travers l'indicateur $T s_{50 \%}$ correspondant au temps nécessaire pour transporter $50 \%$ du flux de MES, on détermine le caractère épisodique du transport de MES : la moitié des sédiments sont transités en $2 \%$ du temps. Ce temps (soit sept jours au cours d'une année) correspond à quelques évènements majeurs les plus productifs en MES. Les résultats obtenus affirment que notre bassin a une dynamique irrégulière vis-à-vis le transport en MES, avec $36 \%$ à $94 \%$ des MES exportés pendant $1 \%$ du temps et $99 \%$ pendant une durée comprise entre $25 \%$ et $50 \%$. Ces résultats sont similaires à certaines études qui montrent que plus de $50 \%$ de la quantité en MES annuelle se produit pendant $1 \%$ du temps (MEADE et al., 1990), durant $2 \%$ du temps (MANO, 2008) et pendant une durée variable entre $1 \%$ et $14 \%$ du temps 
selon les années (VONGVIXAY, 2012). Un total de $90 \%$ de MES transitent pendant une durée comprise entre $13 \%$ et $66 \%$ (VONGVIXAY, 2012), $25 \%$ du temps (OLD et al., 2003 ) et $60 \%$ de MES pendant $10 \%$ du temps (ASSELMAN, 2000). Par ailleurs, d'autres bassins versants ont une dynamique plus irrégulière; ils présentent de faibles valeurs des temps de transfert de MES, avec $75 \%$ de sédiments exportés pendant $1 \%$ du temps et $99 \%$ pendant $6 \%$ pour les bassins de petite superficie (LAGUIONIE, 2007).

Lallure des courbes de la figure 6 peut être modifiée par la situation hydrologique à laquelle est soumis l'ensemble du bassin chaque année, et ce, même avec un débit annuel identique. Dans le cas de l'année 1997, la courbe est représentée par une forme étalée, car les crues sont étalées sur plusieurs jours de pluie et sont régulièrement réparties lors de la saison des pluies provoquant un transport en sédiment moins important. Dans le cas de l'année 2001, la courbe est représentée par une forme convexe, car les crues sont déclenchées par de violents orages d'automne mobilisant des masses importantes de sédiments en très peu de temps.

La part des flux hydriques mensuelle transitée en deux jours les plus contributifs au cours d'un mois varie entre $2 \%$ et $49 \%$ de flux liquide total et comprise entre $10 \%$ et $85 \%$ en 15 jours (Figure 7). Tandis que ces pourcentages sont plus élevés pour les flux de MES : la part des sédiments transportée en deux jours les plus productifs varie entre $8 \%$ et $74 \%$ de flux de MES total, et entre $27 \%$ et $94 \%$ en 15 jours (Figure 8 ). Par conséquent, les jours les plus contributifs en flux liquide et en MES surviennent en mois d'octobre et août, alors que les jours les moins productifs en flux surviennent en décembre.
3.3.2 Mise en évidence des événements les plus intenses: les flux cumulés

Pour mettre en relief le caractère événementiel du transport en suspension, nous avons appliqué les courbes des débits cumulés sur une année de mesure humide et année de mesure sèche (Figure 9). Ces courbes présentent une forme caractéristique en escalier : le flux cumulé est presque constant sur une période, puis augmente brutalement pour atteindre un nouveau palier (MANO, 2008). Limpact des crues sur les volumes d'eau et les flux de MES cumulés est également bien visible, marqué par des courbes en forme d'escalier et des paliers bien nets en dehors des périodes de crues. Néanmoins les paliers sont moins nets pour les volumes d'eau, ce qui indique que le flux liquide est moins irrégulier et se produit tout au long de l'année. Compte tenu des figures ci-dessous, on peut affirmer que tous les évènements de crue véhiculent l'essentiel des MES et semblent avoir la même importance dans le cumul des flux quel que soit l'hydraulicité d'une année. Les figures 9 et 10 illustrent parfaitement l'importance d'évènements brefs sur le flux total annuel. À titre d'exemple, durant l'année humide de 1994, on distingue deux évènements particuliers qui contribuent de manière très importante au flux final des $6,2 \times 10^{6}$ t (Figure 9), environ $95 \%$ de flux total, le premier daté du 23 septembre au 3 octobre 1994 (23e au 33e jour) et le deuxième du 9 au 19 octobre (39e au 49e), dont la durée totale est de 22 jours (soit $6 \%$ du temps). Lors de la crue de mai 2004 (année sèche), environ $0,6 \times 10^{6}$ t ont été transportées en neuf jours (soit $2 \%$ du temps), ce qui représente environ $75 \%$ des $0,8 \times 10^{6}$ t cumulées pour ce bassin versant sur la période 2004-2005 (Figure 10). Ces deux exemples certifient bien l'importance que peuvent prendre quelques évènements les plus intenses de transport en suspension dans le bilan sédimentaire. Le reste des sédiments provient généralement des crues solides hivernales moins violentes.

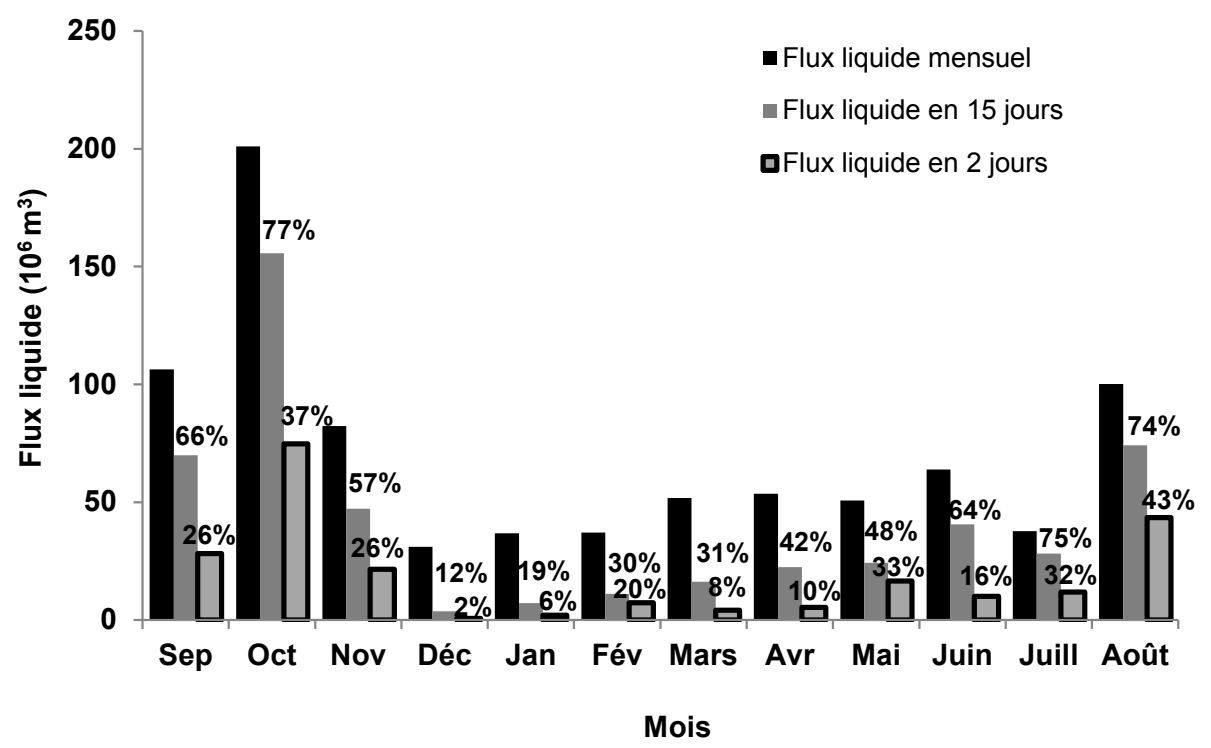

Figure 7. Flux liquides transportés par mois, en 15 jours et en 2 jours. Stream flow transported by month, in 15 days, and in 2 days. 


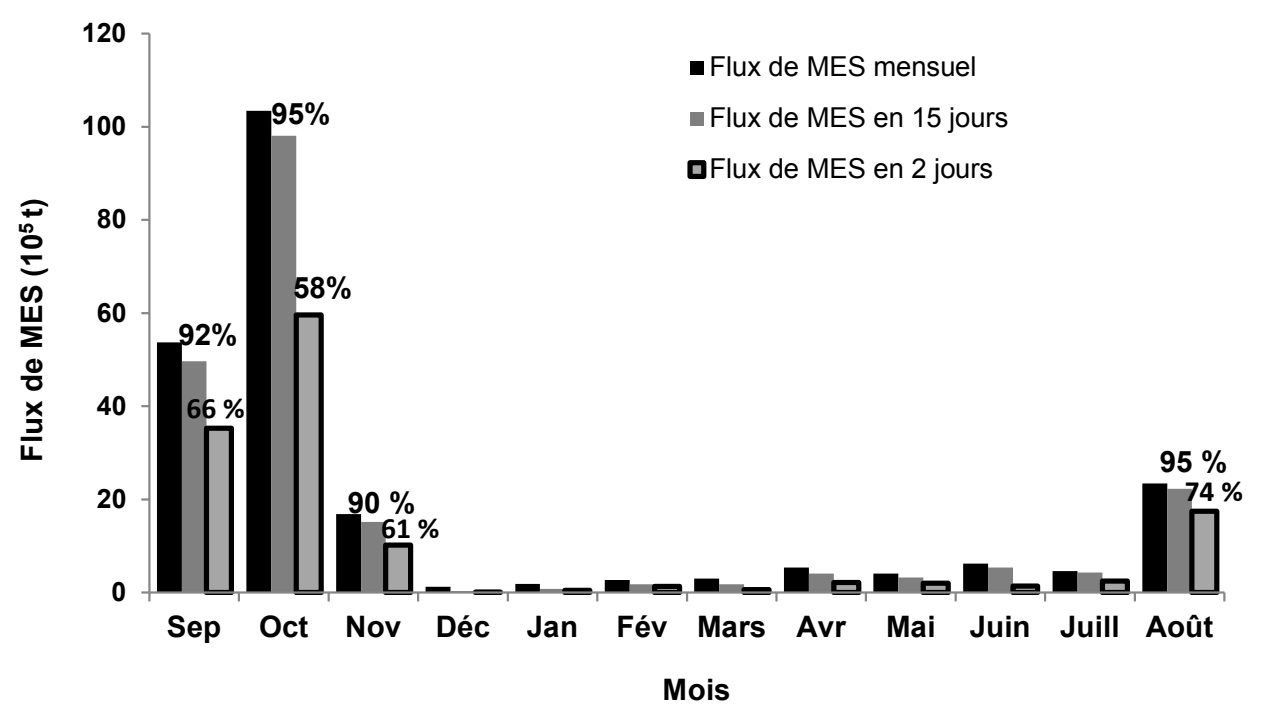

Figure 8. Flux de matières en suspension (MES) transportés par mois, en 15 jours et en 2 jours. Suspended sediment yields transported by month, in 15 days, and in 2 days.

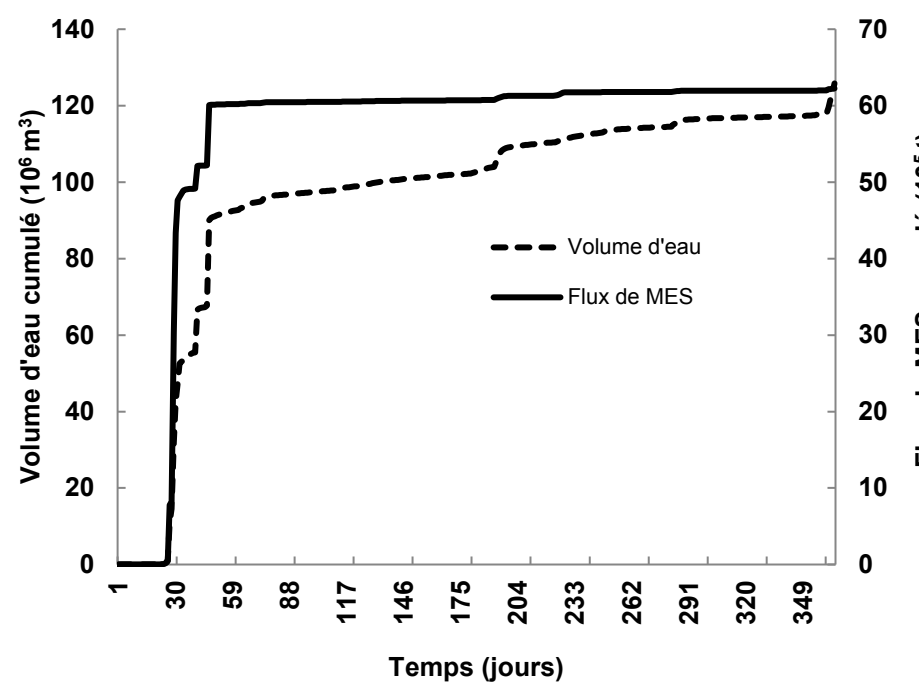

Figure 9. Évolution des flux de sédiments et des volumes d'eau cumulés au cours d'une année humide 1994-1995 sur le bassin de Bou-Hanifia.

Evolution of sediment yield and water volumes accumulated over a wet year (1994-1995) in the Bou-Hanifia Basin.

\section{CONCLUSION}

La dégradation spécifique du sol dans le bassin de BouHanifia calculé à la station des Trois Rivières est de l'ordre $343 \mathrm{t} \cdot \mathrm{a}^{-1} \cdot \mathrm{km}^{-2}$. Cette valeur est dans la gamme des valeurs estimées pour les bassins versants du Maghreb. Cependant, il est nécessaire d'élaborer des mesures de protections antiérosives orientés à :

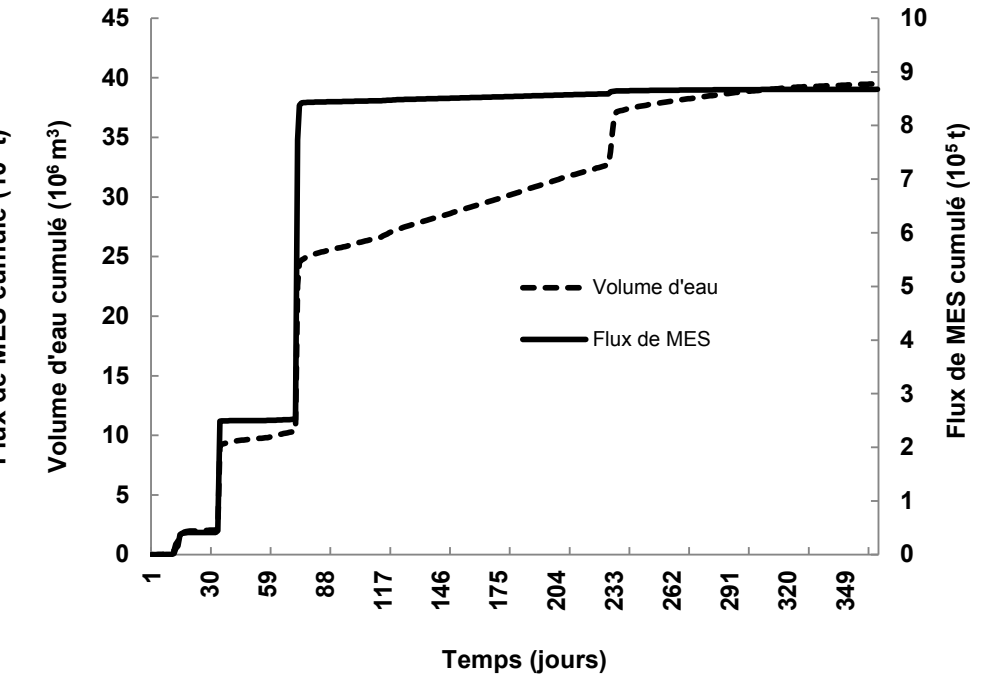

Figure 10. Évolution des flux de sédiments et des volumes d'eau cumulés au cours d'une année sèche (2004-2005) sur le bassin de Bou-Hanifia.

Evolution of sediment yield and water volumes accumulated over a dry year (2004-2005) in the Bou-Hanifia Basin.

i. une meilleure conservation des sols sur le bassin versant dominant la retenue;

ii. la réduction du taux de transport des sédiments vers le barrage et leur envasement. Par conséquent, la prolongation de la durée de vie du barrage aura un impact sur le développement durable des ressources en eau et se répercutera sur l'ensemble des activités économiques liées à la disponibilité de l'eau en quantité et en qualité suffisante. 
Sur un bassin versant comme celui de Bou-Hanifia, le transport en MES ne se produit pas toute l'année, il est plus élevé en automne. Cette répartition irrégulière existe même pour les flux liquides, mais elle est moins marquée. Entre $36 \%$ et $94 \%$ des sédiments sont mobilisés en $1 \%$ du temps (soit trois jours par an) notamment en période de crue.

De l'automne à l'hiver, voire même, jusqu'au printemps, les flux de MES diminuent fortement avec les flux liquides. Ce qui traduit la diminution de la proportion des particules disponibles non renouvelées du fait de la modération de la pluie et la protection plus ou moins progressive des sols par la végétation. Les crues d'hiver sont moins exportatrices de MES, elles contribuent plutôt à l'alimentation de la nappe souterraine qu'au ruissellement. Tandis que les crues qui mobilisent le plus de MES se produisent en fin d'été - début d'automne. La forte augmentation de la quantité de MES observée pendant le mois d'octobre pourrait être due à des conditions favorables à l'érosion, liées à l'absence de la végétation, à des conditions climatiques extrêmes et à l'existence d'un stock important de sédiments dans le bassin versant.

\section{REMERCIEMENTS}

Nous remercions les différents organismes qui nous ont mis à disposition des données de pluie, débit et MES. Les auteurs remercient très sincèrement les réviseurs anonymes de la Revue pour leurs lectures attentives et leurs conseils utiles.

\section{RÉFÉRENCES BIBLIOGRAPHIQUES}

ACHITE M. et M. MEDDI (2005). Variabilité spatiotemporelle des apports liquides et solides en zone semiaride. Cas du bassin versant de l'Oued Mina (Nord-Ouest algérien). Rev. Sci. Eau, 18, 37-56.

ACHITE M. et S. OUILLON (2007). Suspended sediment transport in a semi-arid watershed, Wadi Abd, Algeria (1973-1995). J. Hydrol., 343, 187-202.

ASSELMAN N.E.M. (2000). Fitting and interpretation of sediment rating curves. J. Hydrol., 234 (3-4), 228-248.

BERGAOUI M., H. CAMUS, J.F. NOUVELOT (1998). Essai de modélisation du transport solide sur les microbassins versants de Tebaga (Tunisie Centrale). Sécheresse, 9 (1), 51-57.
BOUANANI A. (2004). Hydrologie, transport solide et modélisation, étude de quelques sous-bassins de la Tafna (Nord-Ouest Algérie). Thèse de doctorat, Univ. Abou Bekr Belkaid, Tlemcen, Algérie, 250 p.

BOUDJADJA A., M. MESSAHEL et H. PAUC (2003). Ressources hydriques en Algérie du Nord. Rev. Sci. Eau, 16 (3), 285-304.

BOUROUBA M. (1998). Phénomène de transport solide dans les Hauts Plateaux Orientaux. Cas de l'Oued Logmane et oued Leham dans le bassin de la Hodna. Rev. Sci. Technol., 9, 5-11.

CHEBBANI R., K. DJILLI et E. ROOSE (1999). Étude à différentes échelles des risques d'érosion dans le bassin versant de l'Isser, Algérie. Bull. Réseau Érosion, 19, 85-95.

EL MAHI A., M. MEDDI et J.P. BRAVARD (2012). Analyse du transport solide en suspension dans le bassin versant de l'Oued El Hammam (Algérie du Nord). Hydrolog. Sci. J., 57 (8), 1-20.

GHENIM A., A. SEDDINI et A. TERFOUS (2008). Variation temporelle de la dégradation spécifique du bassin versant de l'Oued Mouilah (nord-ouest algérien). Hydrolog. Sci. J., 53 (2), 448-456.

HASBAIA M., A. HEDJAZI et L. BENAYADA (2012). Variabilité de l'érosion hydrique dans le bassin du Hodna : cas du sous-bassin versant de l'oued Elham. Rev. Mar. Sci. Agron. Vét., 1, 28-32.

HEUSCH B. et A. MILLIERES- LACROIX (1971). Une méthode pour estimer l'écoulement et l'érosion dans un bassin. Application au Maghreb. Mines et Géologie (Rabat), 33, 21-39.

LAHLOU A. (1988). Étude actualisée de l'envasement des barrages au Maroc. Rev. Sci. Eau, 6 (3), 337-356.

LAGUIONIE P. (2007). Mesures in situ et modélisation du transport des sédiments en rivière. Application au bassin versant de la Vilaine. Thèse de doctorat, Univ. Rennes 1, France, $331 \mathrm{p}$.

LEKFIR A., A. BENKACI et N. DECHEMI (2006). Quantification du transport solide par la technique floue, application au barrage de Beni Amrane (Algérie). Rev. Sci. Eau, 19 (3), 247-257.

LUDWIG W. et J.L. PROBST (1998). River sediment discharge to the oceans: present-day controls and global budgets. Am. J. Sci., 298, 265-295. 
MILLIMAN J.D. et R.H. MEADE (1983). World-wide delivery of river sediment to the oceans. J. Geol., 91, 1-21.

MANO V. (2008). Processus conditionnant les apports de sédiments fins dans les retenues-optimisation des méthodes de mesure et modélisation statistique. Thèse de doctorat, Univ. Joseph Fourier, Grenoble 1, France, 341p.

MEADE R.H., T.R. YUZYK et T.J. DAY (1990). Movement and storage of sediment in rivers of the United States and Canada. Dans: The Geology of North America. Volume 1. Surface Water Hydrology. M.G. WOLMAN et H.C. RIGGS (Éditeurs), Boulder, Geological Society of America, ÉtatsUnis, pp. 255-280.

MEGUENNI K. et B. REMINI (2008). Évaluation du débit solide dans le bassin versant de Harreza (Algérie). Larhyss J., 7, 7-19.

MEGNOUNIF A., A. TERFOUS et A. BOUANANI (2003). Production and transport of suspended sediment in the Upper-Tafna river basin (North West Algeria). Rev. Sci. Eau, 16 (3), 369-380.

MERZOUKI T. (1992). Diagnostic de l'envasement des grands barrages marocains. Recommandations et journée d'étude sur l'érosion du sol et envasement des barrages. Rev. Maroc. Génie Civil, 38, 42-55.

MEYBECK M., L. LAROCHE, H.H. DURR et J.P.M. SYVITSKI (2003). Global variability of daily total suspended solids and their fluxes in rivers. Global Planet. Change, 39 (1-2), 65-93.

MORGAN R.P.C. (1995). Soil erosion and conservation. $2^{\mathrm{e}}$ édition, Longman, Londres, Royaume-Uni, 227 p.

OLD G.H., G.J.L. LEEKS, J.C. PACKMAN, B.P.G. SMITH, S. LEWIS, E.J. HEWITT, H.M. HOLMES et A. YOUNG (2003). The impact of a convectional summer rainfall event on river flow and fine sediment transport in highly urbanized catchment: Bradford, West Yorkshire. Sci. Total Environ., 314-416, 495-512.

PICOUET C., B. HINGRAY et J.O. OLIVRY (2001). Empirical and conceptual modeling of the suspended sediment dynamics in large tropical African rivers: The upper Niger River basin. J. Hydrol., 250, 13-39.
PROBST J.L. et P. AMIOTTE SUCHET (1992). Fluvial suspended sediment transport and mechanical erosion in the Maghreb (North Africa). Hydrolog. Sci. J., 37 (6), 621637.

REMINI B., C. LEDUC et W. HALLOUCHE (2009). Évolution des grands barrages en régions arides : quelques exemples algériens. Sécheresse, 20 (1), 96-103.

SIBARI H., S. HAIDA et A. AIT FORA (2001). Typologie des crues et érosion mécanique dans un bassin versant de zone semi-aride : bassin versant de l'Inaouène, Maroc. Sécheresse, 12, 187-193.

SNOUSSI M. (1988). Nature estimation et comparaison des flux de matière issus des bassins versants de l'Adour (France), du Sebou, de l'Oum-Er-Rbia et du Sous (Maroc). Impact du climat sur les apports fluviatiles à l'océan. Mémoire de l'institut de Géologie du Bassin de l'Aquitaine, No 22, Bordeaux, France, 459 p.

TAVARES J.P. (2010). Érosion des sols au Cap Vert : étude des processus et quantification à l'échelle de trois bassins versants de lîle de Santiago. Thèse de doctorat, Univ. Bourgogne, France, 226 p.

TERFOUS A., A. MEGNOUNIF et A. BOUANANI (2001). Étude du transport solide en suspension dans l'Oued Mouilah (Nord-Ouest algérien). Rev. Sci. Eau, 14, 175185.

VONGVIXAY A. (2012). Mesure et analyse de la dynamique temporelle des flux solides dans les petits bassins versants. Cas d'un bassin versant agricole en région d'élevage (Le Moulinet, Basse-Normandie, France). Thèse de doctorat, INSA de Rennes, France, 185 p.

WALLING D.E. (1984). The sediment yield of African rivers. Dans: Challenges in African hydrology and water resource. Proceedings of the Harare Symposium, juillet 1984, IAHS Publication, No 144, Royaume-Uni, p. 265-283.

WALLING D.E. et B.W. WEBB (1983). Patterns of sediment yield. Dans : Background to paleohydrology. GREGORY K.J. (Éditeur), John Wiley and Sons, Chichester, RoyaumeUni, p. 69-100. 